\title{
Letter
}

\section{Predictors of the Therapeutic Efficacy and Consideration of the Best Combination Therapy of Sodium-Glucose Co-transporter 2 Inhibitors (Diabetes Metab J 2019;43: 158-73)}

\author{
Kyung-Soo Kim \\ Department of Internal Medicine, CHA Bundang Medical Center, CHA University School of Medicine, Seongnam, Korea
}

Metformin is the preferred initial pharmacologic agent for the treatment of type 2 diabetes mellitus (T2DM) unless there are contraindications [1,2]. If the glycosylated hemoglobin (HbAlc) target is not achieved, combination of metformin with other agents should be considered based on drug-specific effects and patient factors $[1,2]$. For patients in whom atherosclerotic cardiovascular disease, heart failure, or chronic kidney disease predominates, the best choice for a second agent is a sodiumglucose cotransporter 2 (SGLT2) inhibitor or glucagon-like peptide 1 receptor agonist (GLP-1 RA), which have been demonstrated to reduce cardiovascular risk [1,2]. Most patients prefer SGLT2 inhibitors because GLP-1 RA needs to be injected. Nonetheless, as SGLT2 inhibitor has gained growing attention in the treatment of T2DM, it is necessary to identify the benefits of using it in combination with other glucose-lowering agents.

In this article entitled "Predictors of the therapeutic efficacy and consideration of the best combination therapy of sodiumglucose co-transporter 2 inhibitors," Lee et al. [3] investigated the predictive markers for the therapeutic efficacy and the best combination strategy using SGLT2 inhibitors in patients with T2DM. After treatment for a median of 192 days with SGLT2 inhibitors, the HbA1c level decreased by $0.7 \%$ (baseline $7.7 \%$ ) and the weight loss was about $3.0 \mathrm{~kg}$. They described that high- er baseline $\mathrm{HbAlc}$ and estimated glomerular filtration rate with a shorter diabetes duration were associated with a better response to SGLT2 inhibitors. Moreover, the add-on of an SGLT2 inhibitor to a dipeptidyl peptidase 4 (DPP4) inhibitor is likely to show the greatest benefit. Interestingly, lower body mass index (BMI) in well-controlled subjects and higher BMI in poorly controlled subjects were associated with better response. However, there are several issues that need to be discussed.

First, this study agrees with previous studies in that obese T2DM patients with inadequate glucose control are more responsive to SGLT2 inhibitors $[4,5]$. On the other hand, there are no studies showing that the lean and well-controlled T2DM patients also associated with better response of SGLT2 inhibitors. Because only 22 patients were selected in the lean and adequately controlled subjects with T2DM, more people are needed for reliable conclusions.

Second, the combination of DPP4 inhibitor and SGLT2 inhibitor is an attractive approach because the two drugs exert complementary glucose-lowering effects and do not induce hypoglycemia. In this study, baseline DPP4 inhibitor users received the greatest benefit from SGLT2 inhibitor therapy. However, Scheen [6] suggested that the additional glucoselowering effect is more marked when a SGLT2 inhibitor is
Corresponding author: Kyung-Soo Kim (D) https://orcid.org/0000-0002-7738-2284 Department of Internal Medicine, CHA Bundang Medical Center, CHA University School of Medicine, 59 Yatap-ro, Bundang-gu, Seongnam 13496, Korea

E-mail: kks982@hanmail.net
This is an Open Access article distributed under the terms of the Creative Commons Attribution Non-Commercial License (http://creativecommons.org/licenses/by-nc/4.0/) which permits unrestricted non-commercial use, distribution, and reproduction in any medium, provided the original work is properly cited. 
added to a DPP4 inhibitor than when a DPP4 inhibitor is added to a SGLT2 inhibitor. The combination of these two drugs showed not additivity for glucose-lowering effect but subadditivity [7]. One possible explanation is that the stimulation of hepatic glucose production by SGLT2 inhibitor-induced glycosuria might be so powerful that it limits the glycemic efficacy of DPP-4 inhibitors in this combination [7].

Finally, any triple combination with SGLT2 inhibitor is not covered by Korean National Health Insurance Service except metformin+sulfonylurea+SGLT2 inhibitor. This might be why metformin+sulfonylurea+SGLT2 inhibitor combination is more common than other triple combination strategies. It is better to consider this situation when interpreting the results of this study.

\section{CONFLICTS OF INTEREST}

No potential conflict of interest relevant to this article was reported.

\section{REFERENCES}

1. American Diabetes Association. 9. Pharmacologic approaches to glycemic treatment: standards of medical care in diabetes: 2019. Diabetes Care 2019;42(Suppl 1):S90-102.

2. Davies MJ, D’Alessio DA, Fradkin J, Kernan WN, Mathieu C,
Mingrone G, Rossing P, Tsapas A, Wexler DJ, Buse JB. Management of hyperglycemia in type 2 diabetes, 2018. A consensus report by the American Diabetes Association (ADA) and the European Association for the Study of Diabetes (EASD). Diabetes Care 2018;41:2669-701.

3. Lee JY, Cho Y, Lee M, Kim YJ, Lee YH, Lee BW, Cha BS, Kang ES. Predictors of the therapeutic efficacy and consideration of the best combination therapy of sodium-glucose co-transporter 2 inhibitors. Diabetes Metab J 2019;43:158-73.

4. Strojek K, Yoon KH, Hruba V, Elze M, Langkilde AM, Parikh S. Effect of dapagliflozin in patients with type 2 diabetes who have inadequate glycaemic control with glimepiride: a randomized, 24-week, double-blind, placebo-controlled trial. Diabetes Obes Metab 2011;13:928-38.

5. Bujac S, Del Parigi A, Sugg J, Grandy S, Liptrot T, Karpefors M, Chamberlain C, Boothman AM. Patient characteristics are not associated with clinically important differential response to dapagliflozin: a staged analysis of phase 3 data. Diabetes Ther 2014;5:471-82.

6. Scheen AJ. DPP-4 inhibitor plus SGLT-2 inhibitor as combination therapy for type 2 diabetes: from rationale to clinical aspects. Expert Opin Drug Metab Toxicol 2016;12:1407-17.

7. van Baar MJB, van Ruiten CC, Muskiet MHA, van Bloemendaal L, IJzerman RG, van Raalte DH. SGLT2 inhibitors in combination therapy: from mechanisms to clinical considerations in type 2 diabetes management. Diabetes Care 2018;41:1543-56. 